\title{
ESCOLHA ADOÇÃO DE TECNOLOGIAS DE INFORMAÇÃO E COMUNICAÇÃO NA EDUCAÇÃO
}

\author{
José Carlos Korelo \\ Mestre em Administração pela Universidade Federal do Paraná - UFPR \\ Pesquisador visitante da Universidade Federal do Paraná - UFPR \\ korelo@yahoo.com

\section{Paulo Henrique Müller Prado} \\ Doutor em Administração de Empresas pela Fundação Getulio Vargas - FGV/SP \\ Professor Adjunto da Universidade Federal do Paraná - UFPR \\ pprado@ufpr.br
}

\section{Danielle Mantovani Lucena da Silva}

Mestre em Administração pela Universidade Federal do Paraná - UFPR

Professora da Faculdade Internacional de Curitiba - FACINTER

dm_lucena@yahoo.com.br

\section{RESUMO}

Nesta pesquisa estuda-se o impacto da escolha sobre a intenção de adoção de novas TICs (tecnologias de informação e comunicação) na educação. O estudo foi realizado através de pesquisa quantitativa, composto de fase exploratória e descritiva. Participaram da fase exploratória 2 diretores (responsáveis pelas TICs) e 4 professores da instituição de ensino superior. $\mathrm{Na}$ fase descritiva quantitativa obteve-se 115 questionários respondidos por professores da instituição analisada. Os dados foram analisados em um modelo de relacionamento multivariado através da modelagem de equações estruturais utilizando-se o método PLS (partial least square). Os resultados dos objetivos de escolha são consistentes com o contexto organizacional (evitar o arrependimento, custos de avaliação, custos de adaptação e evitar emoções negativas) tiveram impacto significativo sobre a tendência a inovar na categoria. Além disso, o estudo mostrou que os professores têm baixa tendência a inovar, sendo que eles utilizam as TICs mais simples na sala de aula.

Palavras-chave: Objetivos de Escolha, Tendência a Inovar, TICs na Educação. 


\section{NTRODUÇÃO}

Estudos sobre difusão de inovações são amplamente discutidos na literatura (e.g. Rogers, 2003; Bass, 1969, Robertson, 1967; Hirshman, 1981; Mahajan; Muller; Bass, 1990; Pádua Jr; Prado, 2005; Goldenberg et al, 2009). Apesar de o assunto estar entre os mais discutidos nas ciências sociais, faz-se necessária uma abordagem específica do tema a respeito dos aspectos relacionados à adoção de novas tecnologias de informação e comunicação (TICs) aplicadas à educação, além da busca por esclarecimentos sobre as características cognitivas dos indivíduos durante o processo de decisão de adoção.

Como a tendência a adotar novos produtos não depende apenas da percepção do indivíduo, mas também do contexto onde este está inserido (Gatignon; Robertson, 1991), tornase relevante a caracterização e escolha adequada do contexto ou categoria de produtos a se estudar. Assim, esta pesquisa se aplica na avaliação da introdução de novas TICs na educação, pois segundo Masetto (2004), esta área é prioritária nas políticas governamentais, tanto brasileiras quanto globais e a UNESCO (Organização das Nações Unidas para a educação, ciência e a cultura) recomenda mudanças que incorporem as novas TICs na educação. Este panorama se deve a necessidade da geração atual de estudantes por novos recursos multimídia e de comunicação, comuns no meio social, alavancados, principalmente por recursos tecnológicos inovadores e pelo uso do computador (Demo, 2005; Nakashima; Amaral, 2006).

Em contrapartida, nem todos os professores são adeptos a essas mudanças. Segundo Belloni (2003), Pretto e Pinto (2006), neste cenário de inovação há os professores deslumbrados, que são totalmente adeptos às novas TICs, os conservadores, que avaliam as novas TICs como negativas, os indiferentes, sem opinião estabelecida e os e sensatos, que avaliam as novas TICs de forma moderada conforme a necessidade. Em virtude dessas diferentes características, a introdução de novas TICs nem sempre pode atingir os resultados esperados pelas IES. Isto se deve ao fato de que apenas o investimento na tecnologia não é suficiente para sua difusão (ROGERS, 2003), é necessário compreender os fatores oriundos da teoria sobre o processo de escolha das mesmas (Bettman; Luce; Payne, 1998), a qual pressupõe que durante a avaliação de alternativas, os indivíduos buscam atingir certos objetivos pré-determinados.

Portanto, o propósito deste estudo é analisar a influência dos objetivos de escolha sobre a tendência a inovar na adoção de tecnologias de informação e comunicação utilizadas por professores. Dado o contexto em análise, os objetivos de escolha considerados no presente estudo são aqueles consistentes com o contexto organizacional (evitar o arrependimento, custos de avaliação, custos de adaptação e evitar emoções negativas) (Luce; Bettman; Payne, 2000; Burnham; Frels; Mahajan, 2003).

Revista de Administração e Inovação, São Paulo, v. 7, n. 2, p. 80-103, abr./jun. 2010 


\section{REVISÃO BIBLIOGRÁFICA}

\subsection{INOVAÇÃO NO CONTEXTO DA EDUCAÇÃO}

A proliferação de equipamentos e dispositivos como MP3, palmtops, celulares, notebooks, datashows e outros ganhou força a partir da junção das indústrias eletrônica, de informática, de entretenimento e comunicação. Isso proporcionou o desenvolvimento de novas tecnologias de informação e comunicação (TIC) na sociedade e no meio educacional (Nakashima; Amaral, 2006). É importante ressaltar que o uso desse termo, tecnologias de informação e comunicação (TIC), é amplo e difuso. Porém, Belloni (2003) o delimita como um conjunto de ferramentas, suportes e canais para o tratamento e acesso à informação que geram novos modos de expressão, novas formas de acesso e novos modelos de participação e recreação cultural.

Então, como as novas TICs proporcionam acesso a informação de forma mais intensa do que as tecnologias anteriores, torna-se conveniente sua utilização na área educacional. A esse fator, somam-se números elevados de computadores e equipamentos que já se encontram instalados nas escolas brasileiras, proporcionando um ambiente favorável para o uso das TICs (MARINHO, 2006).

Porém, o uso das TICs na educação é questionável por diversos autores. Segundo Coutinho e Chaves (2001) as discussões sobre essa temática não são recentes e já vem sendo discutidas há alguns anos. Além disso, esse assunto tem grande relevância no contexto global, onde as políticas governamentais de diversos países têm dado espaço para novas propostas de inovação no ensino. A UNESCO (Organização das Nações Unidas para a educação, ciência e a cultura) possui documentação que preconiza mudanças essenciais inovadoras na educação superior, abordando currículos e métodos que incorporem novas TICs, como educação à distância e a compreensão dos ambientes virtuais (Masseto, 2004).

No Brasil, a educação vem sofrendo profundas alterações, provocadas principalmente pela nova revolução tecnológica da informática e da telemática que, atinge os pilares do processo educacional e da sociedade (Masseto, 2004). Além disso, outros fatores ligados a inovação estão na pauta atual das discussões acadêmicas e o uso de novas TICs na sala de aula é defendido como proposta de tornar o estudante universitário sujeito do processo de aprendizagem, alterando disposições anteriores de cunho meramente reprodutivo, onde o professor entregava todas as informações prontas e sistematizadas para o aluno (Masseto, 2004).

Revista de Administração e Inovação, São Paulo, v. 7, n. 2, p. 80-103, abr./jun. 2010 
O que se percebe é que, rapidamente se intensificaram as pesquisas sobre as inúmeras possibilidades para uso das inovações na educação, merecendo destaque para as possibilidades de uso da educação à distância (EAD), que é considerada por muitos a solução dos problemas de países que ainda lutam com a falta de universalização da educação básica, como é o caso do Brasil. Assim, essas TICs apresentam perspectivas novas, como por exemplo, o respeito à velocidade de aprendizagem individual e a transposição de barreiras, da distância e do tempo. Em contrapartida proporcionam a ocorrência de fraudes e atalhos utilizados por instituições educacionais mercadológicas (Demo, 2005). Para o autor este aspecto é o que proporciona grande força aos críticos à introdução de novas TICs na educação. Entretanto, segundo Demo (2005), não há como escapar da tecnologia na educação, sua introdução e uso são inevitáveis e se esta não for assimilada por bem, será por mau. Isso porque há a crença de que os atuais alunos não têm motivação para a sala de aula, porém os mesmos têm outros tipos de motivações ligados às novas TICs e ao uso incansável do computador.

Moraes, Dias e Fiorentini (2006) argumentam que no Brasil a presença das novas TICs, seja no ensino presencial ou à distância, vem se difundindo rapidamente nos últimos anos, o que obriga as instituições a repensarem o processo de formação de seus professores. Além disso, o ministério da educação preconiza o desenvolvimento de metodologias de ensino que utilizem as novas TICs, pois uma mudança profunda no processo de ensino e aprendizagem somente pode ocorrer se a integração das novas TICs for considerada. Este pensamento do MEC vai de encontro às recomendações da UNESCO e às perspectivas mundiais referentes ao emprego das novas TICs.

Essas perspectivas motivam a analise dos professores quanto ao uso das tecnologias. Drucker (2000) afirma que as novas TICs e a perspectiva da aprendizagem contínua, têm criado novas demandas sociais, exigindo das instituições respostas inovadoras. Dessa forma, intensifica-se o trabalho do professor, já que a escola e todo o sistema educacional passam a funcionar em múltiplos espaços, operando com possibilidades diferentes proporcionadas pelos recursos das novas TICs (Masseto, 2004).

Para esta pesquisa foram analisadas as principais TICs, que dinamizam o ambiente de ensino e aprendizagem, a saber a virtualização, utilizada para ampliação de fontes de consulta através da internet (Nonato, 2007) e os recursos áudio visuais utilizados dentro da sala de aula como datashow e a lousa eletrônica (Nakashima; Amaral, 2006). 


\subsubsection{Tendência a Inovar e Intensidade de Adoção}

Um conceito relevante na literatura de difusão de inovações é o de "innovativeness", que pode ser traduzido como inovatividade ou tendência a inovar. Para Rogers (2003, p. 22), inovatividade "É o grau no qual um indivíduo ou outra unidade de adoção adota novas idéias relativamente mais cedo do que os outros membros de um sistema”. Para o autor, esse construto pode ser medido com base no tempo de adoção, ou seja, indivíduos que adotam inicialmente tendem a ser considerados mais inovadores.

Demais estudos, a partir da década de 90, têm proposto medidas da tendência a inovar de indivíduos em domínios específicos, de uma maneira semelhante a que é utilizada para medir atitudes, estilos de vida e personalidade. Uma dessas propostas é feita por Goldsmith em seus estudos (Goldsmith; Hofacker, 1991; Goldsmith; Flynn, 1992; 1993), que remete a conceitos de curiosidade, de como o indivíduo aceita informações sobre a inovação e como o indivíduo busca essas informações. A escala desenvolvida pelos autores possibilita a mensuração desse construto antes mesmo da utilização de uma tecnologia, pois não foca na mesma, mas sim no comportamento do indivíduo na determinada categoria de produto (e.g. moda; alimentação; tecnologia) em relação a comportamentos regulares de compra ou utilização.

Outra forma de mensuração é a intensidade de posse ou de utilização por parte dos indivíduos. Assim indivíduos que utilizam recursos mais avançados de uma TIC tendem a ser mais inovadores. Para Midgley e Dowling (1978) essa técnica de mensuração pode ser implementada determinando-se quantos itens ou TICs de uma lista da IES o professor utilizou até o tempo da pesquisa. Para identificar o grau de utilização de uma inovação, Marchetti, Prado e Pires (1998), por exemplo, utilizaram uma escala de intensidade do uso de funcionalidades das inovações oferecidas por instituições bancárias. Esta escala foi criada a partir dos estudos de Bagozzi (1995), onde foi utilizada a análise de correspondentes múltiplos (MCA), procedimento HOMALS (SPSS, 1993), para a criação de um escore de pontuação conforme a intensidade de utilização do grupo de inovações desde o mais alto (maior escore) até o mais baixo (menor escore), para classificar os tipos de inovadores.

Nesse sentido, a adoção pode ser medida pela intensidade de utilização das TICs na educação que é um conseqüente do comportamento de tendência a inovar na categoria. Logo, sugere-se a seguinte hipótese: $\mathrm{H}_{1}$ : Quanto maior a tendência a inovar na categoria maior a intensidade de adoção das TICs

Revista de Administração e Inovação, São Paulo, v. 7, n. 2, p. 80-103, abr./jun. 2010 


\subsection{O PAPEL DOS OBJETIVOS DE ESCOLHA NA ADOÇÃO DE NOVAS TIC’S}

Bettman (1979) propôs que os indivíduos possuem uma hierarquia de objetivos, os quais são buscados durante o processo de decisão do produto ou serviço na maioria dos contextos de escolha. Bettman, Luce e Payne (1998, p.192) sugerem que existem quatro objetivos considerados: maximizar a exatidão da escolha, minimizar os custos de avaliação, minimizar a possibilidade de uma experiência negativa advinda da escolha e maximizar a facilidade com que a decisão pode ser justificada. De acordo com os autores, esses objetivos são inerentes a maioria dos contextos de escolha e determinam os principais fatores da análise do processo de decisão.

Heitmann, Lehmann e Herrmann (2007), ainda diferenciam dois tipos de emoções negativas. Os autores propõem que a tentativa de evitar o arrependimento é um dos principais influenciadores da escolha do produto, e é um antecedente das reações emocionais. Dessa forma, este estudo também pressupõe que a tentativa de evitar o arrependimento seja um objetivo de escolha.

Como aborda a definição de Bettman, Luce e Payne (1998), é provável que todos os objetivos influenciem no processo de escolha. Entretanto, dado o contexto organizacional em análise, será avaliada a influência de três desses objetivos sobre a tendência a adotar novas TICs.

O primeiro objetivo analisado é o de "evitar o arrependimento" pela escolha realizada. Os estudos referentes ao arrependimento passaram a ganhar notoriedade após a caracterização de que o processo de tomada de decisão pode ser significativamente influenciado pela capacidade do indivíduo de antecipar sensações de arrependimento, associadas a cada uma das opções disponíveis (Loomes; Sugden, 1982; Tsiros; Mittal, 2000). Esta noção é uma resposta à perspectiva econômica da utilidade esperada, onde uma escolha com certo grau de incerteza é baseada em dois fatores: a utilidade associada à escolha e a probabilidade de ocorrência da escolha, ou seja, trata da tomada de decisão como um processo onde o indivíduo buscará maximizar sua escolha pela utilidade esperada do produto ou serviço que pretende adquirir (Bell, 1982; Pindyck; Rubinfeld, 1994). No contexto educacional, esse objetivo torna-se relevante, pois pode ser mais difícil de ser atingido, caso o indivíduo (professor), tenha menor tendência a inovar.

Ao relacionar o objetivo de evitar o arrependimento com a inovatividade na categoria de produtos, observa-se que em categorias onde é exigido do indivíduo, um conhecimento prévio sobre o produto, como tecnologia, por exemplo, é possível que quanto mais difícil atingir esse objetivo, menor tenda a ser a inovatividade na categoria de produtos.

Revista de Administração e Inovação, São Paulo, v. 7, n. 2, p. 80-103, abr./jun. 2010 
No contexto de adoção de inovação, o receio de se arrepender da escolha pode tornar mais difícil para os indivíduos abrir mão do produto anterior para adotar um novo, pois têm receio de se arrependerem da escolha. Além disso, Wilson e Gilbert (2005) afirmam que os indivíduos têm uma tendência natural para superestimar a intensidade das suas emoções futuras, seja em sentido positivo ou negativo. O comportamento de superestimar as possíveis emoções negativas, dentre elas o arrependimento pode levar os indivíduos à menor tendência a adotar novos produtos. Portanto, supõe-se: $\mathrm{H}_{2}$ : Quanto maior a facilidade de evitar o arrependimento,

\section{maior a tendência a inovar na categoria}

O segundo objetivo analisado refere-se à tentativa de minimizar os custos advindos da escolha. No contexto de novas TICs, o custo de escolha pode ser definido como o custo que um indivíduo associa com o processo de troca de uma TIC por outra nova (Burnham; Frels; Mahajan, 2003). Quando o indivíduo decide que deve trocar uma TIC por outra, mais inovadora, o mesmo pode perceber impedimentos relacionados aos custos de busca de informação, custos de transação, custos de aprendizado, troca de hábitos, custos emocionais e custos de esforço cognitivo, assim como custos financeiros, sociais e psicológico. (Fornell, 1992).

Neste estudo, os principais custos envolvidos na adoção de novas TICs pelos professores são: o custo de avaliação e o custo de adaptação ou aprendizado. O primeiro refere-se ao tempo e o esforço associado à busca e análise para efetuar a decisão de troca (Shugan, 1980; Samuelson; Zeckhauser, 1988). O segundo engloba o tempo e o esforço para aprender novas habilidades ou conhecimento necessário para poder utilizar a nova TIC efetivamente (Alba; Hutchinson, 1987; Eliashberg; Robertson, 1988; Guiltinan, 1989).

Conforme apontado no estudo de Beggs e Klemperer (1992) pode-se supor que os custos de troca tendem a incentivar o professor a continuar utilizando a TIC anterior. Aprender a utilizar um produto novo pode levar tempo e as habilidades exigidas podem não ser assimiladas na sua totalidade, além de haver um custo para o estabelecimento de um relacionamento com o mesmo. Assim pode-se supor que o custo de escolha pode dar à TIC atual um poder monopolista sobre a decisão de troca do indivíduo (Farrell; Shapiro, 1988).

Conforme definiu Hirschman (1980), os novelty seeking ("buscadores de novidade") buscam mais informações e, sobretudo, se interessem em buscá-las. Entretanto, apesar de despenderem mais tempo e recursos, esses consumidores não tendem a perceber tais custos como tão altos em comparação com os consumidores com menor tendência a inovar, pois buscam informações técnicas e direcionadas para o contexto específico de compra.

Revista de Administração e Inovação, São Paulo, v. 7, n. 2, p. 80-103, abr./jun. 2010 
A literatura (Midgley; Dowling, 1978; 1993; Goldsmith; Hofacker, 1991; Roerich, 2004) observa ainda, que a tendência a adotar novos produtos é conseqüência de uma série de atividades direcionadas à busca de novas informações. Neste caso, a percepção do tempo e esforço associados à busca de informações e ao aprendizado das novas habilidades para lidar com o produto pode ser maior para consumidores menos inovadores na categoria.

Como a inovatividade na categoria de produtos propõe que o consumidor tende a adotar as novidades no produto pelo qual se interessa antes da maioria (Goldsmith; Hofacker, 1991), a menor percepção deste custo parece levar a maior tendência a adotar o produto na referida categoria. Portanto: $H_{3 a}$ : Quanto menor a percepção dos custos de avaliação, maior tende a ser a tendência a inovar na categoria; $H_{3 b}$ : Quanto menor a percepção dos custos de adaptação, maior tende a ser a tendência a inovar na categoria.

$\mathrm{O}$ terceiro objetivo refere-se à tentativa de minimizar as possíveis emoções negativas advindas da escolha (Luce; Bettman; Payne, 2000). Shiv, Fedorikhin e Nowlis (2005) argumentam que há grande necessidade de se estudar como os indivíduos tentam lidar com a dificuldade da decisão e seu efeito negativo sobre a escolha final.

As emoções negativas do processo de escolha têm origem nas incertezas que os indivíduos vivenciam tentando alcançar seus objetivos (Greenleaf; Lehman, 1995). A tentativa dos indivíduos de prever as possíveis emoções faz com que alguns consumidores superestimem as possíveis emoções negativas advindas da escolha (Wilson; Gilbert, 2005). Nesse contexto, a menor tendência a adotar novas TICs pode ser conseqüência da tentativa de evitar as emoções negativas advindas da escolha.

Estudos (Lazarus, 1991; Luce; Bettman; Payne, 2000; Herzenstein; Posavac; Brakus, 2007) apontam que existem muitas emoções, tanto positivas quanto negativas, associadas ao processo de escolha de um produto. Entretanto, estudos como os de Mick e Fournier (1998), Pham (1998) e Heitmann, Lehmann e Herrmann (2007) fornecem subsídios para sugerir que o otimismo em relação aos benefícios na inovação, faz os consumidores terem maior tendência a inovar, apesar de também terem certa incerteza sobre o resultado da escolha. Desse modo, podese supor que: $\mathrm{H}_{4}$ : Quanto maior a facilidade de evitar emoções negativas, maior a tendência a inovar na categoria

Tendo a apresentação das hipóteses, o modelo proposto de estudo é uma ampliação dos estudos de Bettman, Luce e Payne (1998), Heitmann, Lehmann e Herrmann (2007) e Midgley e Dowling (1978; 1993), ao agregar os construtos relacionados aos objetivos do consumidor, a tendência a inovar na categoria e a intenção de adoção, conforme observado na figura 1 a seguir:

Revista de Administração e Inovação, São Paulo, v. 7, n. 2, p. 80-103, abr./jun. 2010 


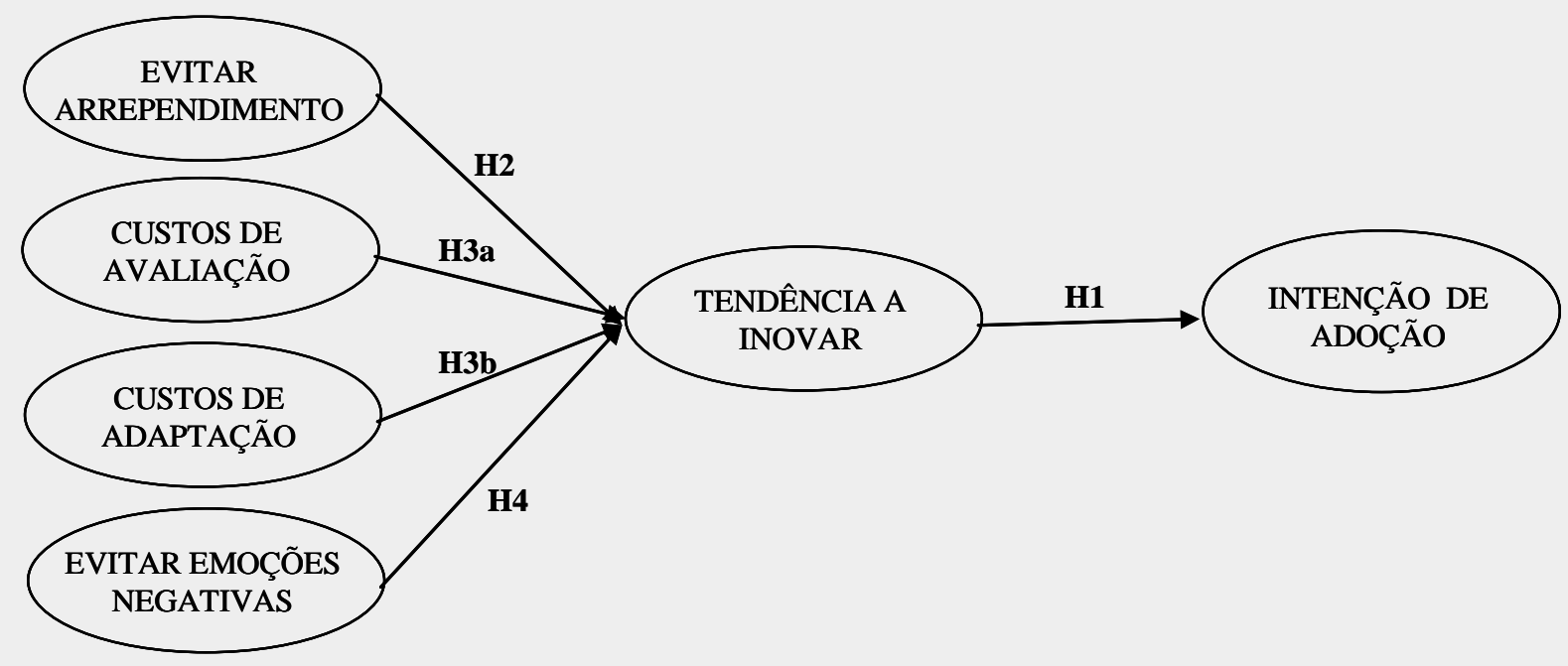

OBJETIVOS DE ESCOLHA

\section{Figura 1: Modelo de estudo proposto}

Fonte: Elaborado pelos autores

\section{METODOLOGIA}

Este estudo, de caráter cross sectional (Malhotra 2006), aplica o método hipotéticodedutivo (Gill; Johnson, 1997). A coleta de dados foi composta de duas fases distintas. A primeira, de caráter exploratório, teve o objetivo compreender as relações e validar os conceitos propostos e a segunda foi quantitativa, do tipo descritiva (Churchill, 1999; Malhotra, 2006).

$\mathrm{Na}$ fase exploratória, as entrevistas foram realizadas com 2 diretores da IES, responsáveis pela introdução ou autorização das novas TICs na organização. As duas entrevistas tiveram a finalidade de prover quais TICs a IES utiliza e quais seriam avaliadas no estudo. Em seguida, 4 professores foram entrevistados com a finalidade de adaptar as escalas propostas na teoria ao contexto em questão. Para este segundo grupo foram selecionados professores com perfis diferentes em relação à afinidade com as novas TICs (1 professor sem afinidade, 2 professores com afinidade moderada e 1 professor com afinidade alta). A escolha dos 4 professores assim como do seu perfil se deu por indicação da administração da IES. Assim o critério de seleção se deu por acessibilidade (Malhotra, 2006).

A fase descritiva, quantitativa foi realizada por levantamento tipo survey, através de questionário estruturado, conforme escalas propostas pela literatura para mensurar os construtos do modelo. Antes da coleta de dados foi realizada a validação das escalas adaptadas ao contexto dos professores por 3 especialistas ( 2 da área de comportamento do consumidor e 1 da área de 
novas TICs na educação) conforme recomenda Hair et al (2005). A aplicação da pesquisa foi feita via internet, com um link disponibilizado aos professores da IES e via papel, ambos auto preenchidos.

Os professores que compuseram a população desta pesquisa lecionam no nível superior, apenas graduação. Para a amostragem, a técnica utilizada foi não probabilística simples, com amostragem restrita aos professores da IES que se foi disponibilizado (MALHOTRA, 2006). Dos 350 professores da população, 120 responderam ao questionário aplicado, 108 via internet e 12 via papel. Após avaliação inicial e eliminação por valores em branco, foram considerados 115 questionários válidos.

Para operacionalizar a mensuração das variáveis ao contexto analisado, foram adaptadas a este estudo as escalas de Evitar o Arrependimento (Loomes; Sugden, 1982; Tsiros; Mittal, 2000; Heitmann; Lehmann; Herrmann, 2007), Custos de Avaliação e Adaptação (Burnham; Frels; Mahajan, 2003; Heitmann; Lehmann; Herrmann, 2007), Evitar emoções negativas (Mick; Fournier, 1998; Luce; Bettman; Payne, 2000) e Tendência a Inovar (Goldsmith; Hofacker, 1991; Goldsmith; Flynn, 1992; 1993). A intenção de adoção foi mensurada pela utilização de produtos na categoria analisada (Bagozzi, 1995).

Para as análises das variáveis utilizaram-se técnicas univariadas e multivariadas com modelagem de equações estruturais (Hair et al, 2005; Oliveira, 2006). Para a estimação de equações estruturais optou-se pelo método dos mínimos quadrados parciais (PLS - partial least square) devido às características de tamanho restrito da amostra (Oliveira, 2006). Além disso, a intensidade de adoção foi avaliada através do procedimento Princals da análise de correspondentes múltiplos (MCA), proposta por Bagozzi (1995) e utilizada por Marchetti, Prado e Pires (1998). Para aplicação destes métodos, contou-se com o auxílio dos softwares SPSS e SmartPLS e Excel.

\section{RESULTADOS}

$\mathrm{Na}$ fase exploratória foram encontradas as TICs elencadas e utilizadas pela IES, a saber: data show (apresentação com textos/tópicos, apresentação com figuras e apresentação com vídeos/animações), laboratório de informática (editor de textos, planilhas e internet), portal IES (sistema de notas, sistema de chamada eletrônica, plano de ensino, conteúdo para alunos, lista de email turma, fórum de debates e avaliação institucional/outras), lousa eletrônica (slides com texto/tópicos, com figuras, com vídeos/animações, com uso de internet e vídeos do youtube e

Revista de Administração e Inovação, São Paulo, v. 7, n. 2, p. 80-103, abr./jun. 2010 
apresentação com uso de convênios como bibliotecas de conteúdo, imagem ou vídeo), tele sala (lousa eletrônica, aulas à distância em tempo real e palestras e treinamentos). Estas TICs compuseram a escala de mensuração do construto intensidade de adoção.

A avaliação também mostrou que a IES estava passando por um processo de venda da estrutura organizacional para outro grupo de ensino nacional e que os professores poderiam se sentir restringidos a responder a pesquisa de forma efetiva.

A análise da fase quantitativa submeteu os resultados a três etapas: (1) preparação da base de dados, onde foram realizadas a análise univariada e multivariada (conferência de médias, limites, desvios padrão, curtose e assimetria, normalidade, linearidade e colinearidade); (2) verificação do modelo de mensuração através de análise fatorial exploratória a consistência interna de cada dimensão, definida pelo alpha de cronbach, e a análise fatorial confirmatória para estabelecer a validade convergente e discriminante de cada construto do modelo; (3) verificação do modelo estrutural proposto por meio de equações estruturais e análise das hipóteses de pesquisa.

\subsection{CARACTERIZAÇÃO DA AMOSTRA}

Do total de 350 professores que compuseram a população desta pesquisa, 115 questionários foram considerados válidos, totalizando $32,8 \%$, destes $74 \%$ estão na faixa de idade de entre 30 e 50 anos. O tempo de atuação na profissão de professor mostrou distribuição de $40 \%$ até 5 anos, 23,5\% entre 6 e 10 anos, 28,7\% entre 11 e 20 anos e 7,8\% acima de 20 anos. Já o tempo de atuação na IES mostrou que aproximadamente metade da amostra (50\%) tem menos de três anos na instituição. A distribuição dos professores da amostra por área de formação apresentou 33,9\% com origem nas Ciências Biológicas e Ciências da Saúde, 16,5\% Ciencias Exatas e Tecnológicas, 28,7\% Ciências Humanas e 20,9\% Ciências Sociais Aplicadas. Por fim, quanto a titulação, $0,9 \%$ dos professores possui somente graduação, $21,1 \%$ possuem especialização, 63,2\% têm mestrado, 12,3\% com doutorado e 2,6\% possui pós-doutorado.

A avaliação descritiva da amostra mostrou valores de média superiores a cinco para todos os indicadores do objetivo evitar o arrependimento e valores de média próximos a quatro para todos os indicadores do objetivo minimizar os custos de avaliação. Esses valores foram considerados em uma escala de sete pontos, mostrando que os professores tendem a não se preocupar com o resultado da escolha que fazem e que os mesmos não avaliam adequadamente os resultados consequentes dessa escolha.

Revista de Administração e Inovação, São Paulo, v. 7, n. 2, p. 80-103, abr./jun. 2010 
Os custos de aprendizado e adaptação também apresentaram média próxima a quatro, o que pode caracterizar um comportamento de indiferença por parte dos professores, já que na escala de sete pontos esses valor é o ponto mediano entre discordo totalmente e concordo totalmente.

O objetivo evitar emoções negativas apresentou media alta para todos os seus indicadores, acima de cinco, mostrando que os professores não acham que o processo de troca gera emoções negativas. A avaliação da tendência a inovar nas TICs mostrou que todos os indicadores apresentaram média ligeiramente superior a quatro, mostrando concordância pequena com as proposições de mensuração do construto e, ainda, dois itens apresentaram média abaixo de três, confirmando essa baixa avaliação da tendência a inovar.

\subsection{VERIFICAÇÃO DO MODELO DE MENSURAÇÃO}

O resultado da análise fatorial exploratória constatou validade na unidimensionalidade dos quatro objetivos de escolha propostos, sendo evitar o arrependimento (alpha $=0,667$, variância explicada $=61,59 \%$ e KMO $=0,663$ ), custos de escolha de avaliação (alpha $=0,797$, variância explicada $=71,42 \%$ e KMO $=0,612)$, custos de escolha de aprendizado (alpha = 0,719, variância explicada $=64,14 \%$ e KMO $=0,676$ ), evitar emoções negativas (alpha $=0,901$, variância explicada $=66,29 \%$ e KMO $=0,851$ ). Para o construto tendência a inovar nas TICs também foi comprovada a unidimensionalidade, porém dos 9 indicadores iniciais, 1 foi eliminado por não contribuir com a dimensão, restando 8. Seguem-se os valores da análise $($ alpha $=0,771$, variância explicada $=63,71 \%$ e KMO $=0,721)$.

Já o construto intensidade de adoção foi avaliado, em termos de dimensionalidade e composição de escala, através da análise de correspondentes múltiplos (MCA). Das cinco TICs definidas inicialmente na fase exploratória para compor a escala, duas foram eliminadas por não estarem efetivamente disponível para a adoção até a coleta dos dados, restando o data show, o laboratório de informática e o portal da IES.

Os resultados proporcionaram agrupamento das funcionalidades das TICs em duas categorias ou dimensões, consideradas como TICs utilizadas dentro da sala de aula e fora da sala de aula, devido à suas características. As TICs agrupadas na dimensão dentro da sala de aula foram o data show e o laboratório de informática e as agrupadas fora da sala de aula foram os recursos do portal da IES.

Revista de Administração e Inovação, São Paulo, v. 7, n. 2, p. 80-103, abr./jun. 2010 
Essa inspeção também proporcionou a geração de um escore que permitiu a ordenação dos professores em função da intensidade de adoção. A confiabilidade da dimensão TICs dentro da sala de aula foi (alpha $=0,883$ ) e TICs fora da sala de aula foi (alpha $=0,871)$. Nesta última dimensão que representa a TIC portal IES, os recursos conteúdo para alunos, lista de email turma e fórum de debates foram eliminados por não contribuírem com a dimensão. Ao final dos dessa fase dos 49 indicadores iniciais restaram 36.

A análise de validade dos construtos avaliou a validade convergente e discriminante. A validade convergente pode ser verificada na tabela 1 e observou como primeiro critério os carregamentos dos indicadores em seus respectivos construtos, sendo que a variável latente evitar arrependimento apresentou um indicador não adequado, enquanto a variável tendência a inovar apresentou dois indicadores fora de padrão. Estes foram eliminados do modelo de mensuração e o modelo foi recalculado novamente.

Os demais critérios de avaliação da validade convergente foram a análise da variância média extraída (AVE), que apresentou valores satisfatórios para todas as dimensões, a confiabilidade composta (CONF), que também garantiu números satisfatórios e o teste de significância t bootstrapping do método PLS que apresentou valores maiores que 1,96 (BOEHE, 2005) para todos os itens, exceto para as dimensões dentro da sala e fora da sala, que apresentaram valores 1,000 correspondente a apenas 1 fator no construto. Isto foi efetuado, pois dois novos construtos que substituem o construto intensidade de adoção foram considerados, já que os fatores dentro da sala de aula e fora da sala de aula mensuram TICs diferentes, que a análise de correspondentes sugeriu. Os valores encontrados na tabela 1 atestam a validade convergente do modelo proposto, ou seja, os grupos de indicadores estão convergindo e medindo os respectivos construtos.

A validade discriminante foi analisada por três critérios, o primeiro comparou os carregamentos dos indicadores e fatores em seus respectivos construtos com os carregamentos cruzados, ou em outros construtos para verificar se algum indicador poderia obter carga maior em outro construto. Foi verificado que em nenhum construto houve carga de outro indicador maior do que os próprios indicadores do construto em análise. O segundo critério foi a verificação da correlação entre as variáveis latentes do modelo, sendo que essas não podem ser superior a 0,95 (HAIR et al, 2005). Todas as correlações analisadas apresentaram valores inferiores.

Revista de Administração e Inovação, São Paulo, v. 7, n. 2, p. 80-103, abr./jun. 2010 
Tabela 1: Validade convergente do modelo de mensuração

\begin{tabular}{|c|c|c|c|c|c|c|c|}
\hline INDICADORES/ CONSTRUTOS & 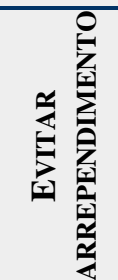 & 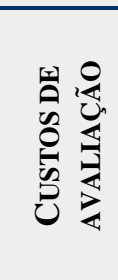 & 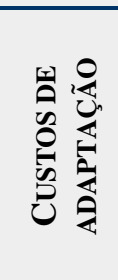 & 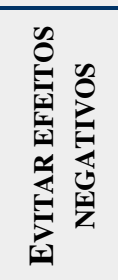 & 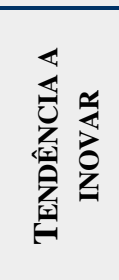 & 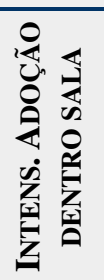 & 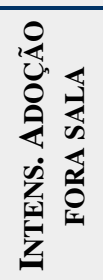 \\
\hline Não repensa em decisões & $0,980^{*}$ & & & & & & \\
\hline Fez a melhor escolha & $0,667 *$ & & & & & & \\
\hline Não dispõe de tempo & & $0,879 *$ & & & & & \\
\hline Comparações exigem tempo & & $0,916^{*}$ & & & & & \\
\hline É difícil comparar aplicações & & $0,834^{*}$ & & & & & \\
\hline Aprender leva tempo & & & $0,864 *$ & & & & \\
\hline Esforço p/ aprender pequeno & & & $0,847 *$ & & & & \\
\hline A adaptação é desagradável & & & $0,912 *$ & & & & \\
\hline Envergonhado & & & & $0,906^{*}$ & & & \\
\hline Angustiado & & & & $0,906^{*}$ & & & \\
\hline Triste & & & & $0,951^{*}$ & & & \\
\hline Irritado & & & & $0,924 *$ & & & \\
\hline Nervoso & & & & $0,928^{*}$ & & & \\
\hline Tenso & & & & $0,929 *$ & & & \\
\hline Com medo & & & & $0,940^{*}$ & & & \\
\hline Culpado & & & & $0,928 *$ & & & \\
\hline Hostil & & & & $0,945^{*}$ & & & \\
\hline Assustado & & & & $0,961 *$ & & & \\
\hline Último a usar no meio social & & & & & $0,797 *$ & & \\
\hline Interesse em usar & & & & & $0,749 *$ & & \\
\hline Utilização pequena comparado & & & & & $0,759 *$ & & \\
\hline Geralmente lê periódicos & & & & & $0,670 *$ & & \\
\hline Acessa portais especializ. & & & & & $0,691 *$ & & \\
\hline Lê menos comparado ao meio & & & & & $0,612 *$ & & \\
\hline Dentro sala & & & & & & 1,000 & \\
\hline Fora sala & & & & & & & 1,000 \\
\hline AVE & 0,703 & 0,770 & 0,776 & 0,869 & 0,513 & 1,000 & 1,000 \\
\hline Conf. Composta & 0,821 & 0,909 & 0,907 & 0,985 & 0,862 & 1,000 & 1,000 \\
\hline
\end{tabular}

* teste de significância bootstrapping $\mathrm{t}>1,96$

Fonte: Análise de dados coletados

O último critério de validade discriminante foi a verificação do quadrado das correlações entre as variáveis. Para que sejam adequados os resultados devem ser inferiores aos índices de AVE para cada construto. Os valores dos quadrados das correlações não foram superior às AVEs das variáveis latentes. Assim os critérios de avaliação de validade discriminante atestaram que não houve sobreposição de variáveis. 
Ao final da avaliação da análise fatorial confirmatória dos 36 indicadores que iniciaram o processo 33 foram considerados adequados aos construtos do modelo de mensuração. Assim, se pode avançar para os testes do modelo proposto e das suas hipóteses.

\subsection{VERIFICAÇÃO DO MODELO ESTRUTURAL E ANÁLISE DAS HIPÓTESES}

A análise do modelo estrutural foi realizada através de duas etapas. A primeira avaliou os coeficientes de regressão (caminhos) entre os construtos e a significância dos mesmos (valor t). Os resultados apontam relevância entre as seguintes relações: evitar arrependimento e tendência a inovar (coef $=0164$, valor $t=3,032)$, evitar efeitos negativos e tendência a inovar (coef $=$ 0,239 , valor $\mathrm{t}=5,064$ ) e tendência a inovar e intensidade de adoção dentro da sala (coef $=0,319$, valor $\mathrm{t}=4,108$ ). As demais relações não obtiveram relevância, sendo elas: minimizar custos de avaliação e tendência a inovar (coef $=0,108$, valor $\mathrm{t}=1,681$ ), minimizar custos de adaptação e tendência a inovar (coef $=0,094$, valor $t=1,378)$ e tendência a inovar e intensidade de adoção fora da sala $($ coef $=-0,046$, valor $\mathrm{t}=0,855)$.

A segunda etapa de avaliação do modelo estrutural foi a conferência da variância explicada das variáveis dependentes do modelo. As variáveis dependentes são a tendência a inovar, intensidade de adoção dentro da sala e intensidade de adoção fora da sala. A variância explicada pode ser estimada através do quadrado das múltiplas correlações $\left(\mathrm{R}^{2}\right)$ e esta é a medida de qual proporção de que uma variável dependente é explicada pelas antecedentes (HAIR et al, 2005). Os resultados da avaliação do $\mathrm{R}^{2}$ mostram que $22,7 \%$ da tendência a inovar é explicado pelos objetivos de escolha, mostrando uma relação baixa. Quanto a intensidade de adoção dentro da sala $10,2 \%$ é explicado pela tendência a inovar e a intensidade de adoção fora da sala é explicada em apenas $0,2 \%$ pela tendência a inovar, mostrando uma interação muito fraca nessa última relação e considerada inválida, menor do que 10\%, como estipulado por (BOEHE, 2005).

Após avaliação do modelo estrutural, foi realizada a análise das hipóteses propostas. A tabela 2 apresenta a avaliação das hipóteses e as relações entre as variáveis que a sustentam (regressão). Para essa análise a hipótese $\mathrm{H} 1$ ficou subdividida em $\mathrm{H}_{1 \mathrm{a}}$ que relaciona a tendência a inovar e a intensidade de adoção dentro da sala de aula e $\mathrm{H}_{1 \mathrm{~b}}$ que relaciona a tendência a inovar na categoria com a intensidade de adoção fora da sala de aula. Esta divisão segue a segmentação proposta pela construção da escala na análise de correspondentes realizada na verificação do modelo de mensuração.

Revista de Administração e Inovação, São Paulo, v. 7, n. 2, p. 80-103, abr./jun. 2010 
Os resultados apontaram que a hipótese $\mathrm{H}_{1 \mathrm{a}}$ foi confirmada, mostrando que a tendência a inovar na categoria influencia positivamente a intensidade de adoção das TICs utilizadas dentro da sala de aula. Entretanto hipótese $\mathrm{H}_{1 b}$ não foi confirmada, mostrando que a tendência a inovar não apresentou influência na intensidade de adoção das TICs utilizadas fora da sala de aula.

\section{Tabela 2: Avaliação de Hipóteses}

\begin{tabular}{|l|l|c|c|c|}
\hline HIP. & \multicolumn{1}{|c|}{ RELAÇãO ESTRUTURAL } & COEF. & VAL T & VERIFICAÇão \\
\hline H1a & $\begin{array}{l}\text { Tendência a inovar na categoria e intensidade de adoção das } \\
\text { TICs utilizadas dentro da sala de aula }\end{array}$ & 0,319 & $4,108^{*}$ & confirmada \\
\hline H1b & $\begin{array}{l}\text { Tendência a inovar na categoria e intensidade de adoção das } \\
\text { TICs utilizadas fora da sala de aula }\end{array}$ & $-0,046$ & 0,855 & não confirmada \\
\hline H2 & $\begin{array}{l}\text { Facilidade de evitar arrependimento e tendência a inovar na } \\
\text { categoria }\end{array}$ & 0,164 & $3,032^{*}$ & confirmada \\
\hline H3a & $\begin{array}{l}\text { Percepção dos custos de avaliação e tendência a inovar na } \\
\text { categoria }\end{array}$ & 0,108 & 1,681 & não confirmada \\
\hline H3b & $\begin{array}{l}\text { Percepção dos custos de adaptação e tendência a inovar na } \\
\text { categoria }\end{array}$ & 0,094 & 1,378 & não confirmada \\
\hline H4 & $\begin{array}{l}\text { Facilidade de evitar emoções negativas e tendência a inovar na } \\
\text { categoria }\end{array}$ & 0,239 & $5,064^{*}$ & confirmada \\
\hline
\end{tabular}

* teste de significância bootstrapping $\mathrm{t}>1,96$

FONTE: Elaborado pelos autores

A hipótese $\mathrm{H}_{2}$ foi confirmada pelo modelo, assim a facilidade de evitar o arrependimento influencia positivamente a tendência a inovar na categoria. A hipótese $\mathrm{H}_{3 a}$ não obteve confirmação, assim como a hipótese $\mathrm{H}_{3 b}$, o que implica que a percepção dos custos de avaliação e adaptação são maiores por parte dos professores, não impactando de forma significativa a tendência a inovar na categoria. Por fim, a hipótese $\mathrm{H}_{4}$ foi confirmada, o que implica que a facilidade de evitar emoções negativas impactou positivamente a tendência a inovar na categoria.

\section{DISCUSSÃO DOS RESULTADOS}

Os resultados verificados na relação proposta em $\mathrm{H}_{1 \mathrm{a}}$ e $\mathrm{H}_{1 \mathrm{~b}}$ são consistentes com a teoria de adoção de inovações. No primeiro caso, a confirmação da hipótese $\left(\mathrm{H}_{1 \mathrm{a}}\right)$ é conseqüência da proposta teórica dos estudos de Goldsmith (GOLDSMITH; HOFACKER; 1991; GOLDSMITH; FLYNN, 1992), a qual sugere que os indivíduos tendem a ser inovadores em uma determinada categoria e não em outra. Portanto, como professores, é possível que o contexto profissional no qual atuam os faça ter maior intensidade de adoção dentro da sala de aula. Em contrapartida, a não confirmação da $\mathrm{H}_{1 b}$, que relaciona a tendência a inovar na categoria com a intensidade de adoção fora da sala de aula pode estar relacionada à menor tendência a inovar nessa categoria. 
A confirmação da relação proposta em $\mathrm{H}_{2}$ é plausível tanto sob a ótica teórica quanto prática. Se os professores tentam evitar o erro de adotar uma nova TIC e falharem, serão avaliados de forma negativa pela IES e pelos alunos. Porém, conforme apontaram as entrevistas da fase qualitativa, os acadêmicos tendem a aceitar com tranquilidade que o professor utilize novos aplicativos em sala de aula. Isso tende a minimizar a possibilidade de o professor se arrepender ao adotar tal tecnologia.

No contexto de adoção de inovação, o receio de se arrepender da escolha pode tornar mais difícil para os consumidores abrir mão do produto anterior para adotar um novo, pois têm receio de se arrependerem da escolha (Wilson; Gilbert, 2005). Porém, no caso em análise, a instituição coloca as tecnologias à disposição dos professores, e por isso, torna-se mais fácil trocar uma tecnologia antiga pela nova.

Além disso, as análises das hipóteses $\mathrm{H}_{3 \mathrm{a}}$ e $\mathrm{H}_{3 \mathrm{~b}}$ evidenciaram que a percepção dos professores quanto aos custos de aprendizado e adaptação não são minimizados o suficiente para resultar em uma relação estatisticamente significativa sobre a tendência a adotar, o que tende a tornar mais difícil a adaptação às novas TICs, sob esse aspecto. Isso implica que mesmo a instituição disponibilizando a tecnologia, o professor pode avaliar como altos os custos de aprender a usar tais recursos e se adaptar aos mesmos. Este, portanto, parece ser um objetivo mais difícil de ser atingido pelos professores e tende a dificultar a tendência a inovar.

A literatura (Midgley; Dowling, 1978; 1993; Goldsmith; Hofacker, 1991; Roerich, 2004; Goldenberg et al, 2009) observa ainda que a tendência a adotar novos produtos é consequiência de uma série de atividades direcionadas à busca de novas informações. Neste caso, a percepção do tempo e esforço associados à busca de informações e ao aprendizado das novas habilidades para lidar com o produto pode ser maior para consumidores menos inovadores na categoria.

A hipótese $\mathrm{H}_{4}$ foi confirmada no presente estudo, o que implica que a facilidade de adoção de novas TICs parece estar muito mais relacionada a aspectos cognitivos do comportamento individual do que propriamente às emoções. Tais evidências são verificadas não apenas pela confirmação desta última hipótese, mas também pela confirmação da $\mathrm{H}_{2}$ e pela não confirmação das hipóteses $\mathrm{H}_{3 \mathrm{a}}$ e $\mathrm{H}_{3 \mathrm{~b}}$.

A resistência à adoção de novas TICs parece estar basicamente relacionada aos custos de abrir mão de um equipamento ainda visto como útil para o professor para aprender a utilizar um novo equipamento. Conforme sugerido no estudo de Beggs e Klemperer (1992), os custos de troca tendem a incentivar o professor a continuar utilizando a TIC anterior. 
Os resultados obtidos na relação entre os objetivos de escolha e a tendência a inovar podem ser explicados também ao se analisar a relação proposta em $\mathrm{H}_{1 \mathrm{a}}$ e $\mathrm{H}_{1 \mathrm{~b}}$. Em relação à intensidade de adoção, a avaliação do modelo mostrou que os professores utilizam as TICs dentro da sala de aula com mais intensidade, porém isso ocorre com os recursos mais básicos, verificado na avaliação da escala de adoção. Já entre as TICs utilizadas fora da sala de aula, como é o caso do portal da IES, somente os recursos obrigatórios ou determinados pela IES são utilizados, porém de forma geral esse construto não apresentou valores elevados na escala de adoção. Estes indícios mostram que, na maioria, os professores parecem apresentar menor tendência a inovar, pois utilizam o que é simples ou o que é solicitado pela IES. Isto pode ser confirmado pelo baixo poder de explicação das variáveis dependentes de intensidade de adoção no modelo estrutural.

\section{CONSIDERAÇÕES FINAIS}

Uma contribuição relevante dessa pesquisa foi a aplicação da teoria dos objetivos de escolha (Bettman; Luce; Payne, 1998) em um contexto organizacional e de novas TICs na educação, caracterizado como ambiente de inovação, onde segundo Bettman, Luce e Payne (1998), o número de atributos pode ser considerado alto, dificultando o processo de decisão. Assim, os resultados mostraram que o comportamento apresentado pelos professores reflete dificuldade em adotar novas TICs na IES. Isto se deve ao fato de que os professores não conseguem visualizar as vantagens da adoção das mesmas em um futuro próximo, tanto para si quanto para a IES, proporcionando pela dificuldade de avaliar os custos envolvidos e dificuldade de evitar as emoções negativas advindas da troca da técnica atual pela nova.

Este tipo de estudo também pode ser considerado relevante, pois segundo Masseto (2004), nas pesquisas em educação essas propostas têm grande importância, já que para a incorporação das novas TICs é necessário desenvolvimento de metodologias adequadas e isso somente pode ser realizado se, dentre outros fatores os professores forem bem avaliados em relação ao grau de utilização das novas TICs, além de compreenderem o seu papel na mudança ocasionada por utilizar uma nova TIC.

Deve-se destacar algumas limitações do estudo, como as decorrentes da escolha metodológica, já denotadas pela literatura (Malhotra, 2006), a qual argumenta que o tipo de pesquisa descritiva através de survey não consegue profundidade na explicação dos resultados. Então apesar do modelo mostrar que as relações propostas entre as variáveis são válidas, ele é superficial na obtenção dos motivos.

Revista de Administração e Inovação, São Paulo, v. 7, n. 2, p. 80-103, abr./jun. 2010 
Outro fator limitante da pesquisa foi a dificuldade em atingir o número de 350 professores, que caracterizava a população. Somente 115 questionários foram considerados válidos de 120 participantes. Assim, o poder de explicação do modelo se restringiu ao tamanho da amostra. Com 115 casos válidos não foi possível utilizar no modelo de equações estruturais alguns modelos complementares por subgrupo de amostra (e.g. idade, nível de especialização, etc) para verificar a curva de adotantes proposta por Rogers (2003). Além disso, foi utilizado método de analise de equações estruturais PLS (partial least square), para amostras não normais ou pequenas. Este método é questionado por ter força de explicação limitada, diminuindo a capacidade de explicação do modelo.

O que se sugere em pesquisas futuras, é aplicação do questionário para uma amostra mais significativa do que a realizada, com o intuito de aumentar a possibilidade do desenvolvimento de modelos complementares ao proposto. Isto tornaria possível a comparação das hipóteses entre os diversos tipos de adotantes caracterizados na curva de inovadores proposta por Rogers (2003), proporcionaria uma possível taxonomia de professores adotantes de TICs, facilitando o trabalho da IES no emprego dessas ferramentas.

Também se tornaria interessante aumentar o número de novas TICs que não foram possíveis de analisar nessa pesquisa, como a lousa eletrônica e as ferramentas de ensino à distância como tele sala. Ligado a esse fator, a introdução de fatores exógenos que as IES praticam, poderiam ajudar a aumentar o poder de explicação dos fenômenos ligados a adoção de novas TICs por professores e fornecer respostas de avaliação desses fatores para sua maximização ou eliminação.

Por fim, um método de pesquisa mais aprofundado (qualitativo) que considere questões organizacionais poderia agregar relevância e entendimento a fatores não explicados por essa estratégia de pesquisa empregada.

\section{REFERÊNCIAS BIBLIOGRÁFICAS}

Alba, J.W; Hutchinson, J.W. Dimensions of consumer expertise. Journal of Consumer Research, 13, 411-54, March 1987.

Bagozzi, R. Advanced methods of marketing research. Oxford: Blackwell, 1995.

Bass, F.M. A new product growth for model consumer durables. Management Science. 15 (5) 215-27, 1969.

Revista de Administração e Inovação, São Paulo, v. 7, n. 2, p. 80-103, abr./jun. 2010 
Beggs, A.; Klemperer, P. Multi-Period competition with switching costs. Econometrica, 60, 3, 651-66, May 1992

Bell, D.E. Regret in decision making under uncertainty. Operations Research, 30, 961-81, 1982.

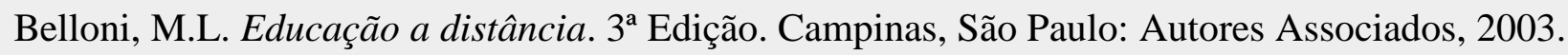

Bettman, J.R. An information processing theory of consumer choice. Cambridge, MA: AssisonWesley, 1979.

Bettman, J.R.; Luce, M.F.; Payne, J.W. Constructive consumer choice processes. Journal of Consumer Research, 25, 187-214, dec. 1998.

Boehe, D. Desenvolvimento de produtos em subsidiárias de empresas multinacionais no Brasil. Tese de Doutorado. Universidade de São Paulo. São Paulo, 2005.

Burnham, T.A., Frels, J.K.; Mahajan, V. A typology, antecedents, and consequences. Academy of Marketing Science Journal, 31, 2, 109-26, Spring, 2003.

Churchill, J.R.; G.A. Marketing research: methodological foundations, 7th Ed. Fort Worth: Dryden, 1999.

Coutinho, C.M.G.F.P; Chaves, J.H.S. Desafios à Investigação em TIC na Educação: As Metodologias de Desenvolvimento. II Conferência Internacional Challenges 2001/ Desafios 2001.

Demo, P. Éticas Multiculturais - Sobre convivência humana possível. Vozes: Petrópolis, 2005.

Drucker, P.A nova sociedade das organizações. In: HOWARD, R. (Org.) Aprendizado organizacional. Rio de Janeiro: Campus, 2000. p.1-7.

Eliashberg, J.; Robertson, T.S. New product preannouncing behavior: a market signaling study. Journal of Marketing Research, 25, 282-92, Aug, 1988.

Farrell, J.; Shapiro, C. Dynamic competition with switching costs. The Rand Journal of Economics, 19, 1, 123-37, Spring 1988.

Fornell, C. A national customer satisfaction barometer: the Swedish experience. Journal of Marketing 56, 1, 6-21, Jan. 1992.

Gatignon, H.; Robertson, T. S. Innovative decision processes. In: ROBERTSON, T. S.; KASSARJIAN, H. H. Handbook of consumer behavior. New Jersey: Prentice-Hall, 316-48, 1991.

Gill, J.; Johnson, P. Research methods for managers. 2ed. London: Sage, 1997.

Revista de Administração e Inovação, São Paulo, v. 7, n. 2, p. 80-103, abr./jun. 2010 
Goldenberg, J.; Han, S.; Lehmann, D.R.; Hong, J. The role of hubs in the adoption process. Journal of Marketing, 73, 2, 1-13, mar. 2009.

Goldsmith, R.E.; Hofacker, C. F. Measuring consumer innovativeness. Journal of the Academy of Marketing Science, 19, 3, 209-21, jun. 1991.

Goldsmith, R.E.; Flynn, L.R. A validation of the Goldsmith and Hofacker innovativeness scale. Educational and Psychological Measurement, 53, 4, 1105-16, Winter, 1993.

Goldsmith, R.E.; Flynn, L.R. Identifying innovators in consumer product markets. European Journal of Marketing, 26, 12, 42-55, 1992.

Greenleaf, E.A.; Lehmann, D.R. Reasons for substantial delay in consumer decision making. Journal of Consumer Research, 22, 2, 186-99, set.1995.

Guiltinan, J.P. A classification of switching costs with implications for relationship marketing, AMA Winter Educators' Conference: Marketing Theory and Practice, 216-20, 1989

Hair Jr, J.F.; Babin, B.; Money, A.; Samouel, P. Fundamentos de métodos de Pesquisa em Administração. Porto Alegre: Bookman, 2005.

Heitmann, M.; Lehmann, D.R.; Herrmann, A. Choice goal attainment and decision consumption satisfaction. Journal of Marketing Research, 44, 234-50, may 2007.

Herzenstein, M.; Posavac, S.S.; Brakus, J. J. Adoption of new and really new products: the effects of self regulation systems and risk salience. Journal of Marketing Research, 44, 2 , 251-60, may 2007.

Hirschman, E. C. Innovativeness, novelty seeking, and consumer creativity. Journal of Consumer Research, 7, 283-95, dec. 1980.

Hirschman, E. Technology and symbolism as sources for the generation of innovation. Advances in Consumer Research, 9, 537-41, 1981.

Lazarus, R. S. Emotion and adaptation. New York: Oxford University Press, 1991.

Loomes, G.; Sugden, R. Regret theory: an alternative theory of rational choice under uncertainty. The Economic Journal, 92, dec. 1982.

Luce, M.F.; Bettman, J.R.; Payne, J.W. Minimizing negative emotion as a decision goal: investigating emotional trade-off difficulty. In: Ratneshwar, S.; Mick.; Huffman, C. The why of consumption: contemporary perspectives on consumer motives, goals and desires. London: Routdedge, 2000.

Mahajan, V.; Muller, E.; Bass, F.M. New product diffusion models in marketing: a review and directions for research. Journal of Marketing. 54, 1-26, jan. 1990. 
Malhotra, N.K. Pesquisa de marketing: uma orientação aplicada. 4a . Ed. Porto Alegre: Bookman, 2006.

Marchetti, R.Z.; Prado, P.H.M.; Pires, P.J. Análise de correspondentes múltiplos aplicada à ordenação de dados qualitativos. XXII Enanpad, Foz do Iguaçu, 1998.

Marinho, S.P.P. Novas tecnologias e velhos currículos; já é hora de sincronizar. Revista ECurriculum, 2, 3, 35-38, dec. 2006.

Masetto, M. Innovation in higher education. Interface - Comunicação, Saúde, Educação, 8, 14, set.2003-fev.2004.

Mick, D.G., Fournier, S. Paradoxes of technology: consumer cognizance, emotions, and coping strategies. Journal of Consumer Research, 25, 123-43, 1998.

Midgley, D.F.; Dowling, G.R. Innovativeness: the conception and its measurements. Journal of Consumer Research, 4, 229-42, mar. 1978.

Moraes, R.A.; Dias, A.C.; Fiorentini, L.M.R. As tecnologias da informação e comunicação na educação: as perspectivas de Freire e Bakhtin. UNI Revista, 1, 3, julho 2006.

Nakashima, R.H.R.; Amaral, S.F. A linguagem audiovisual da lousa digital interativa no contexto educacional. ETD - Educação Temática Digital, Campinas, 8, 1, 33-50, dez. 2006.

Nonato, E.R.S. EAD, TIC e Internet: Ainda Estranhas à Escola. $13^{\circ}$ Congresso Internacional de Educação a Distância, Curitiba, PR, set 2007.

Oliveira, D. Mensurando o valor da marca, a reputação e a identidade no setor automotivo. Dissertação em Administração pela UFMG. Belo Horizonte, 2006.

Pádua Júnior, F. P.; Prado, P. H. A adoção de inovações em produtos de alta tecnologia por jovens: o caso do telefone celular. In: Anais do XXIX ENANPAD, Brasília, 2005.

Pham, M.T. Representativeness, relevance and the use of feelings in decision making. Journal of Consumer Research, 25, 144-57, sept. 1998.

Pindyck, R.; Rubinfeld, D. Microeconomia. São Paulo: Makron Books, 1994.

Pretto, N.; Pinto, C.C. Tecnologias e novas educações. Revista Brasileira de Educação, 11, 31 jan./abr. 2006.

Robertson, T.S. The process of innovation and the diffusion of innovation. Journal of Marketing, 31, 14-19, jan. 1967.

Roehrich, G. Consumer innovativeness: concepts and measurements. Journal of Business Research, 57, 671-77, 2004.

Revista de Administração e Inovação, São Paulo, v. 7, n. 2, p. 80-103, abr./jun. 2010 
Rogers, E.M. Diffusion of innovations. 5. ed. New York: Free Press, 2003.

Samuelson, W.; Zeckhauser, R. Status quo bias in decision making. Journal of Risk and Uncertainty, 1, 7-59, 1988

Shiv, B.; Fedorikhin, A.; Nowlis, M.N. Interplay of the heart and the mind in decision-making. In: Ratneshwar, S.; Mick, D. G. Inside consumption: consumer motives, goals and desires. Routledge: New Jersey, 2005.

Shugan, S.M. The costs of thinking. Journal of Consumer Research 7, 99-111, September 1980.

Tsiros, M.; Mittal, V. Regret: a model of its antecedents and consequences in consumer decision making. Journal of Consumer Research, 26, 4, 401-17, mar.2000.

Wilson, T.D.; Gilbert, D. T. Affective forecasting: knowing what to want. Current Directions in Psychological Science, 14, 3, 131-34, 2005.

\title{
SELECTION AND ADOPTION OF INFORMATION AND COMMUNICATION TECHNOLOGIES IN EDUCATION
}

\begin{abstract}
This research studied the impact of choice of using new ICTs (information and communication technologies) in education. The study was performed through quantitative research, composed of exploratory and descriptive sections. Two directors responsible for ICTs and four higher education teachers participated in the exploratory phase. In the quantitative descriptive phase questionnaires were administered to 115 teachers of higher education. The data were analyzed in a multivariate relationship model through structural equation modeling using the PLS method (partial least squares regression). The results showed the objects of choice was consistent with the context of the organization which is to avoid regret, assessment costs, compliance costs and negative emotions. This had a significant impact about the trend to innovate in the category. The study showed that the teachers have a low tendency to innovate when they only use simple ICTs in the classroom.
\end{abstract}

Key-words: Objectives of Choice, Tendency to Innovate, ICTs in education.

Data do recebimento do artigo: 20/05/2009

Data do aceite de publicação: 11/03/2010

Revista de Administração e Inovação, São Paulo, v. 7, n. 2, p. 80-103, abr./jun. 2010 